\title{
A Comparison of Lower Limb Peripherally Inserted Central Catheter and Deep Venous Catheterisation
}

\author{
Feng-Xian Li, Yu-Fen Hao, Mei-Jing Tian, Yan-Ping Li and Hong-Yang Zhang \\ Department of Oncology, Beijing Shijingshan Hospital, Capital Medical University, Beijing, China
}

\begin{abstract}
Objective: To compare the clinical application effects of peripherally inserted central catheter (PICC) and deep venous catheters placed through the lower limbs in adults with malignancy obviating the use of upper limb PICC.

Study Design: Descriptive study.

Place and Duration of Study: Department of Oncology, Beijing Shijingshan Hospital, Capital Medical University, Beijing, China between February 2017 and February 2019.

Methodology: The patients enrolled in this study were assigned to PICC under ultrasound guidance group and deep venous catheterisation group. The inclusion criteria were patients with advanced malignant tumor after bilateral breast cancer lymphadenectomy or superior vena cava obstruction syndrome; bedridden patients; and patients without mental disorders; who could understand the content of this study and agreed to participate in this study. Patients with high-risk thrombosis and venous thrombosis of lower limbs were excluded. The success rate of one-time catheterisation, the length of catheterisation puncture time, the number of catheterisations, and the occurrence of catheter-related complications of the two groups were compared.

Results: Ultrasound-guided PICC through the lower limbs had a higher success rate than deep venous catheterization $(84.6 \%$ vs. $42.9 \%, p=0.046)$. The average length of puncture time in adults with PICC through the lower limbs under ultrasound guidance was shorter than that in the femoral vein group $(24.69 \pm 4.35 \mathrm{vs} .29 .14 \pm 6.02, p=0.038)$. No catheter-related infection was found in the two groups of patients.

Conclusion: The indications for PICC through the lower limbs in adults include patients with advanced malignant tumor after bilateral breast cancer lymphadenectomy or superior vena cava obstruction syndrome, and mainly bedridden patients. This technique has certain advantages over deep venous catheterisation and is an effective choice for venous access.
\end{abstract}

Key Words: Lower limb PICC, Deep venous catheterisation, Advanced malignant tumor, Bedridden.

How to cite this article: Li FX, Hao YF, Tian MJ, Li YP, Zhang HY. A Comparison of Lower Limb Peripherally Inserted Central Catheter and Deep Venous Catheterisation. J Coll Physicians Surg Pak 2021; 31(12):1478-1481.

\section{INTRODUCTION}

Peripheral venous catheterisation, such as cephalic vein and median cubital vein, or catheter tip in the inferior vena cava or superior vena cava, is called peripherally inserted central catheter (PICC). ${ }^{1} \mathrm{PICC}$ has the characteristics of convenience, safety, comfort, and long indwelling time. It has been used increasingly during the treatment of patients with malignant tumor, especially for patients receiving chemotherapy and patients with end-stage malignant tumor.

Correspondence to: Feng-Xian Li, Department of Oncology, Beijing Shijingshan Hospital, Capital Medical University, Beijing, China

E-mail: fengxianlidr@163.com

Received: December 14, 2020; Revised: June 04, 2021;

Accepted: June 30, 2021

DOI: https://doi.org/10.29271/jcpsp.2021.12.1478
The 2016 edition of "Practical Standards for Infusion Therapy" states that PICC puncture veins, including the axillary vein, temporal vein, posterior auricular vein of the head, and great saphenous vein of the lower extremity, can be selected for newborns and children. ${ }^{2-4}$ However, adults also need PICC through lower limbs, such as patients with advanced malignancies after bilateral breast cancer lymphadenectomy and superior vena cava obstruction syndrome.

If there is abnormal appearance of the superior vena cava in the body, which causes it to block the vein, the superior vena cava blood cannot flow smoothly. This leads to other clinical manifestations of the body, such as varicose veins, which is superior vena cava obstruction syndrome. ${ }^{5}$ Lymphedema after breast cancer surgery is due to surgery or radiotherapy that hinders the transport capacity of the lymphatic system, causing the accumulation of lymph fluid in the tissues ${ }^{6,7}$ For patients with superior vena cava obstruction syndrome and bilateral breast cancer after radical mastectomy, to avoid increasing the pressure of the superior vena cava and aggravating the lymphedema of the head, face and upper limbs, only lower limb 
infusion can be used. For chemotherapy administration and nutrition support, the central vein pathway is needed because of the high incidence of phlebitis and fluid infiltration due to repeated puncture of peripheral veins of lower limbs. Therefore, PICC through lower limbs in adults is one of the effective ways to provide intravenous infusion access for such patients.

This study aimed to compare the clinical application effects of peripherally inserted central catheter (PICC) and deep venous catheters placed through the lower limbs in adults with malignancy obviating the use of upper limb PICC.

\section{METHODOLOGY}

This was a descriptive study. Patients with malignancy obviating the use of upper limb PICC in Beijing Shijingshan Hospital, Capital Medical University from February 2017 to February 2019 were enrolled in this study. These patients were assigned to PICC under ultrasound guidance group and deep venous catheterisation group. The study was approved by the Institutional Review Board.

The inclusion criteria were patients with advanced malignant tumor after bilateral breast cancer lymphadenectomy or superior vena cava obstruction syndrome; bedridden patients; and patients without mental disorders so that they could understand the content of this study and agreed to participate in this study. Patients with high-risk thrombosis and venous thrombosis of lower limbs were excluded.

A 4Fr catheter obtained from Bard Access Systems Inc. 605 North 5600 West, Salt Lake City, Utah, 84116, USA was used in the PICC group. Operator: Ultrasound-guided PICC through lower limbs was conducted by experienced nurses with a catheterisation qualification. The modified Seldinger technique was used. Briefly, the puncture needle without needle core was used to puncture the blood vessel directly. When the puncture needle penetrated the anterior wall of the blood vessel and entered the blood vessel, the blood could be seen ejecting from the tail of the needle, and then the guide wire catheter could be introduced. The difference between the modified Seldinger procedure and the classical Seldinger procedure is that the former does not need to penetrate the posterior wall of the blood vessel, so it has high success rate and less complications.

Under ultrasound guidance, the puncture point of PICC was selected as the middle of the thigh. The catheter tip was inserted into the level of the first lumbar spine or the level of the 3-4 lumbar spine, as suggested by previous studies. ${ }^{8,9}$ The estimated catheter insertion length was the sum of the distance from the puncture point to the midpoint of the inguinal ligament, the distance from the midpoint of the inguinal ligament to the umbilicus, and the distance from the umbilicus to the lower edge of the xiphoid process. After completing this operation, the tip position was checked by X-ray.

The maintenance methods of PICC were shown as follows: First, the leg circumference was measured, and the skin around the puncture site was disinfected three times with a $75 \%$ alcohol cotton ball and three times with an iodine cotton ball. Then, $0.9 \%$ normal saline was used to flush the tube and fix it. Similar to the upper limb PICC, the longest indwelling time of lower limb PICC was also one year. ${ }^{10}$

An 8Fr catheter obtained from SCW Medicath Ltd. was used in the lower limb deep vein catheterisation group. As described previously, ${ }^{11}$ the medial side of the femoral vein was selected as the puncture point. The tip was inserted into the superior segment of the inferior vena cava. The insertion length was about $15 \mathrm{~cm}$. After this operation, the tip position was checked by X-ray examination.

The success rate of one-time catheterisation, the length of the catheterisation puncture, the number of catheterisations, and the occurrence of catheter-related complications of the two methods were studied. IBM SPSS Statistics version 25.0 (IBM Inc) was used for the statistical analysis. The qualitative data were given as numbers and percentages, while quantitative as mean $\pm S$.D. The independent sample t-test was used for the age and puncture time of catheterisation. The success rate of one-time catheterisation, the number of catheterisations, and the occurrence of complications related to catheterisation were tested with Chi-square or Fisher's Exact test. $P<0.05$ was considered significant.

\section{RESULTS}

A total of 27 patients were included in this study. Among them, 14 patients were enrolled in the deep vein catheterisation group, with a mean age of $73.36 \pm 10.11$ years; and 13 patients were enrolled in the PICC catheter group with a mean age of $71.85 \pm 14.67$ years. The comparison of general data between the two groups are shown in Tablel.

Table I: Comparison of general data between the two groups.

\begin{tabular}{|l|c|c|c|c|}
\hline \multirow{2}{*}{ Group } & \multirow{2}{*}{ Age } & \multicolumn{2}{|c|}{ Gender } & \multirow{2}{*}{ Case } \\
\cline { 3 - 4 } & & Female & Male & \\
\hline Deep vein catheterization & $73.36 \pm$ & 5 & 9 & 14 \\
group & 10.11 & $(35.7 \%)$ & $(64.3 \%)$ & $(100 \%)$ \\
\hline \multirow{2}{*}{ PICC catheter group } & $71.85 \pm$ & 6 & 7 & 13 \\
& 14.67 & $(46.2 \%)$ & $(53.8 \%)$ & $(100 \%)$ \\
\hline$p$ & 0.757 & \multicolumn{4}{|c|}{0.581} & \\
\hline
\end{tabular}

Table Il: Comparison of observation indices between the two groups.

\begin{tabular}{|l|c|c|c|c|}
\hline Group & $\begin{array}{c}\text { Patients } \\
\text { No. }\end{array}$ & $\begin{array}{c}\text { Success rate } \\
\text { of disposable } \\
\text { catheterization }\end{array}$ & $\begin{array}{c}\text { Average } \\
\text { Catheter } \\
\text { replacement } \\
\text { rate } \\
\text { time } \\
\text { rinutes) } \\
\text { mean } \pm \\
\text { standard } \\
\text { deviation }\end{array}$ \\
\hline $\begin{array}{l}\text { PICC catheter } \\
\text { group }\end{array}$ & 13 & $11(84.6 \%)$ & $0(0 \%)$ & $24.69 \pm 4.35$ \\
\hline $\begin{array}{l}\text { Deep vein } \\
\text { catheterization } \\
\text { group }\end{array}$ & 14 & $6(42.9 \%)$ & $5(35.7 \%)$ & $29.14 \pm 6.02$ \\
\hline$p$ & & 0.046 & 0.041 & 0.038 \\
\hline
\end{tabular}

There was no significant difference in the incidence of catheterrelated infection $[0(0 \%)$ vs. $3(21.4 \%) p=0.222]$, catheter-related deep vein thrombosis $[0(0 \%)$ vs. $2(14.3 \%) p=0.481]$, 
catheter blockage [0(0\%) vs. $1(7.1 \%)$ p >0.999], and unplanned extubation complications between the two groups [0(0\%) vs. $3(21.4 \%) p=0.222$ ].

Results of clinical research showed that success rate of one-time catheterisation in PICC catheter group was significantly higher than that in deep vein catheterisation group $(p=0.046$, Table II). And the average length of puncture time in PICC catheter group was much shorter than that in deep vein catheterisationgroup $(p=0.038$, Table II).

\section{DISCUSSION}

The 2016 edition of the "Practical Standards for Infusion Therapy" recommends neither the insertion of PICC through the lower limbs in adults nor the indication, vascular selection, puncture point location and catheter tip position in PICC through lower limbs for adults. Some domestic scholars ${ }^{9,11,12}$ have conducted research on adult lower limb PICC. There are big differences in the selection of puncture veins and the selection of puncture points. Some studies ${ }^{8}$ have selected puncture sites in the proximal thigh, others ${ }^{9}$ in the middle or upper segment. Some studies ${ }^{11}$ choose great saphenous vein, others ${ }^{13,14}$ choose femoral vein as puncture vessel.

Some studies ${ }^{11,12}$ state that the complications associated with PICC through lower limbs include catheter-related infection, catheter-related thrombosis, catheter blockage, and unplanned extubation. $\mathrm{Li}$ ' $\mathrm{s}{ }^{15}$ study reported that the incidence of deep vein catheter thrombosis was as high as $22.22 \%$, which was significantly higher than that of internal carotid artery and subclavian artery catheterisation. Deep vein thromboembolism might cause pulmonary embolism.

The selected cases in this study are patients with contraindications for upper limb catheterisation, especially the long-term bedridden patients. Since the position of these patients is consistent with the daily body position of infants mentioned in the guidelines (mostly the lying position), they will not cause significant changes in the hemodynamics of lower limbs due to standing or walking frequently, and it is not easy to cause a twisted, folded and ectopic catheter. Therefore, it is suggested that the indications of PICC through lower limbs in adults are limited to those who cannot receive upper limb infusion, especially those who are mainly in a lying position. The femoral vein was chosen as the puncture vein in this study, since owing to its deep position, fast flow rate, and thick diameter, it is not easy for vascular complications to develop, and it is easy to operate. However, the great saphenous vein is small in diameter, has many variations, and has many venous valves, and it is difficult to puncture. The puncture point selected was the middle of the thigh, far away from the perineal area to reduce the incidence of infection complications, and far away from the gathering area of nerves and lymphatic vessels to reduce the incidence of nerve injury and lymphatic leakage.

There is no corresponding standard for the selection of blood vessels and puncture site in adult lower limb PICC catheterisa- tion. In this study, the great saphenous vein was selected, which has a small diameter, many variations, and many venous valves, which are difficult to puncture. Therefore, ultrasound-assisted puncture was used to improve the success rate.

The femoral vein, which is relatively thick in diameter and easy to operate, was chosen as the puncture vein in the deep venous catheterisation group in this study. The PICC insertion operation in the author's hospital was done by nurses, and ultrasound equipment was a necessary auxiliary tool. The deep venous catheterisation is was done by the doctors with bare hands, without the assistance of ultrasound equipment. Therefore, there is no ultrasound auxiliary equipment for deep venous catheterisation in the comparative study of lower limb PICC catheterisation.

Results of this study indicate that compared with deep vein catheterisation of lower limbs, ultrasound-guided PICC through the lower limb in adults has a higher success rate and can be completed in a shorter time. There was no difference in the complications of the two procedures. The characteristics of lower limb PICC are as follows: Long indwelling time; the PICC has a smaller diameter and the material is soft, which makes patients feel more comfortable; the improved Sedinger technique is used during puncture, which causes less trauma; and the use of ultrasound guidance provides a higher success rate.

Therefore, in certain patients, the relevant indications must be fully understood to apply the intravenous treatment method of lower limb PICC safely and effectively. Patients with advanced malignant tumors, who are bedridden and have a limited survival period, it is necessary to give them rescue measures in time and ensure their nutrition. Under this condition, if only a short peripheral venous catheter of the lower limbs is used, the patient's treatment needs cannot be met. Therefore, this study was limited to patients with advanced malignant tumors after bilateral breast cancer lymphadenectomy and superior vena cava obstruction syndrome. Lower limb PICC has become one of the effective intravenous treatment pathways for patients of this type.

\section{CONCLUSION}

The indications for PICC through lower limbs in adults are limited to patients with contraindications for upper limb infusion, such as patients with advanced malignant tumors after bilateral breastcancerlymphadenectomy or superiorvena cava obstruction syndrome, and mainly bedridden patients. Compared with short peripheral venous catheterisation of lower limbs, this method has certain advantages and is one of the venous access options for such patients.

\section{FUNDING:}

This work was supported by Hospital level scientific research in Beijing Shijingshan Hospital in 2018. [No. : sjsky-201811].

\section{ACKNOWLEDGMENT:}

No funding or sponsorship was received for this study or publication of this article. 


\section{ETHICALAPPROVAL:}

This study was conducted in accordance with the Declaration of Helsinki and with the approval from the Ethics Committee of Beijing Shijingshan Hospital, Capital Medical University (LUNSHENKEYAN DI 202009). The ethical approvals were obtained prior to initiation of the research work.

\section{PATIENTS' CONSENT:}

After explaining the operation steps, all patients gave their written informed consent.

\section{CONFLICT OF INTEREST:}

The authors declared no conflict of interests.

\section{AUTHORS' CONTRIBUTION:}

FXL: Substantial contributions to conception and design, acquisition of data, analysis and interpretation of data.

YPL, YFH: Drafted the manuscript and revised it critically for important intellectual content. MJT, HYZ: Final approval of the version to be published.

\section{REFERENCES}

1. Johann DA, De Lazzari LS, Pedrolo E, Mingorance $P$, De Almeida TQR, Danski MTR. Peripherally inserted central catheter care in neonates: An integrative literature review. Rev ESC Enferm USP 2012; 46(6): 1503-11. doi: 10.1590/ s0080-62342012000600030.

2. Dawson RB. PICC Zone Insertion Method ${ }^{\mathrm{TM}}\left(\mathrm{ZIM}^{\mathrm{TM}}\right)$ : $A$ systematic approach to determine the ideal insertion site for PICCs in the upper arm. Journal of the Association for Vascular Access 2011; 16(3):156-60,162-5. doi.org/10.2309/ java.16-3-5.

3. Liem TK, Yanit KE, Moseley SE, Landry GJ, Deloughery TG, Rumwell CA, et al. Peripherally inserted cen-tral catheter usage patterns and associated symptomatic upper extremity venous thrombosis. J Vasc Surg 2012; 55(3): 761-7.doi: 10.1016/j.jvs.2011.10.005.

4. Sharp R, Cummings M, Fielder A, Mikocka-Walus A, Grech $C$, Esterman $A$. The catheter to vein ratio and rates of symptomatic venous thromboembolism inpatients with a peripherally inserted central catheter (PICC): A prospective cohort study. Int J Nurs Stud 2015; 52(3):677-85. doi.org/ 10.1016/j.ijnurstu.2014.12.002.

5. Li JT, Lao YL, Xie YJ. English Chinese Dictionary of clinical syndromes in surgery and obstetrics and Gynecology. China Union Medical University Press 2001:616.
6. Chen R. Clinical study on breast reconstruction and treatment of lymphedema of upper limbs after breast cancer surgery. Peking Union Medical College 2016. doi:10.7666/d.Y3075367.

7. Zhang BN. Prevention strategies and treatment principles of upper limb lymphedema after breast cancer operation. Chinese Journal of Practical Surgery 2015; 35(7):723-7. doi:10.7504/CJPS.ISSN1005-2208.2015.07.09.

8. Kang GQ, Wang JH. The effect of downward shift of insertion site for femoral vein peripherally inserted central catheters in patients with end-stage diseases receiving palliative therapies. Journal of Nursing Science 2018; 33(11):58-9. doi:10.3870/j.issn.10014152.2018.11.058

9. Zhang JH, Li XB, He LX, Cao LM, Zhang CL, Tang SY. The feasibility of ultrasound-guided PICC insertion by the middle-thigh femoral vein in lung cancer patients with superior vena cava obstruction. Chinese J Nursing 201; 50(6):692-6. doi:10.3761/j.issn.0254-1769.2015.06.011.

10. Tang TT, Liu L, Li CX, Li YT, Zhou T, Li HP, et al. Which is better for patients with breast cancer: Totally implanted vascular access devices (TIVAD) or peripherally inserted central catheter (PICC)? World J Surg 2019; 43(9):2245-9. doi: 10.1007/s00268-019-05022-X.

11. Wu XF, Ying LM, Zhan XY, Tan WF. PICC placement via the greater saphenous vein in patients with superior vena cava syndrome. Chinese J General Practice 2018; 16(3): 494-7.doi: 10.16766/j.cnki.issn.1674-4152.000137.

12. Zhang $L$. The clinical effect and safety evaluation of femoral vein PICC catheterization in malignant tumor patients with obstruction of superior vena cava. J Nurses Training 2017; 32(1):72-5.doi:10.16821/j.cnki.hsjx.2017. 01.027.

13. Chan YJ, Li YY, Ye GX. Clinical study on the placement of PICC catheter via femoral vein puncture in 5 patients. J Qilu Nursing 2009; 15(10):15-6. doi:10.3969/j.issn.10067256.2009.10.008.

14. Zhao L. Application and nursing of PICC catheter inserted through femoral vein in critically ill patients in ICU. J Qilu Nursing 2009; 15(10):71-2. doi:10.3969/j.issn.10067256.2009.10.040.

15. Li JH, Wu WD, Huang JH, Zhang L, Shao XW. The analysis of high risk factors of central venous catheter thrombosis in hemodialysis patients. Contemporary Med 2017; 23(18): 3-5. doi:10.3969/j.issn.1009-4393.2017.18.002. 